\title{
Making your mind up
}

\section{Neurons with sustained activity could help us to understand cognition.}

\section{Cortex and Mind: Unifying \\ Cognition \\ by Joaquín M. Fuster \\ Oxford University Press: 2002.314 pp. \\ $\$ 49.95, £ 47.95$}

\section{Kevan Martin}

"Human cognition may like the winged horse take at times its flights toward the stars and forget Earth.” This was Charles Sherrington's own flight of fancy as he pondered the ancient question of the relationship between the working of the brain and the working of the mind in his Gifford lectures of 1937-38. How close are we now to an answer? Joaquín Fuster provides a progress report in Cortex and Mind, in which he considers several aspects of cognition: perception, memory, intelligence, language and attention. What is striking from his account is how much we now rely on data from experimental animals, particularly primates, to understand the physical basis of human cognition - more even than Sherrington, who was perhaps the greatest of all behavioural physiologists.

Those familiar with Fuster's previous book Memory in the Cerebral Cortex (MIT Press, 1994) will know that he discovered in the prefrontal cortex of monkeys a group of neurons that sustain their activity between a sensory cue and a delayed motor response. The existence of such 'working memory' cells had long been predicted, and soon afterwards Hiroaki Niki found a second class, which accelerate their firing over the delay period.

These two classes are intermixed in most of the prefrontal cortex and are also found in parietal cortex. It is not yet clear whether such sustained activity arises through reverberation in the recurrent cortical circuits, as proposed by Lorente de Nó and currently the theorist's favourite, or through mnemonic capabilities at the level of single neurons, as suggested by in vitro experiments by Alexei Egorov and colleagues, and no definitive answer is expected soon. But the question is nevertheless important: how do our cognitive processes map onto the brain's circuits?

For Fuster, this question is decided by his view that the brain is a neural network, currently best represented by connectionist models involving parallel distributed processing. The essential currency of cognition for Fuster is the 'cognit', a word he coins here to describe an item of knowledge that is stored in a network. Each node of the cortical neural network stores yet more elementary representations that make up a cognit. Not all cognits are equal. Symbols, for example, are presented in high-ranking cognits, themselves produced by the convergence of
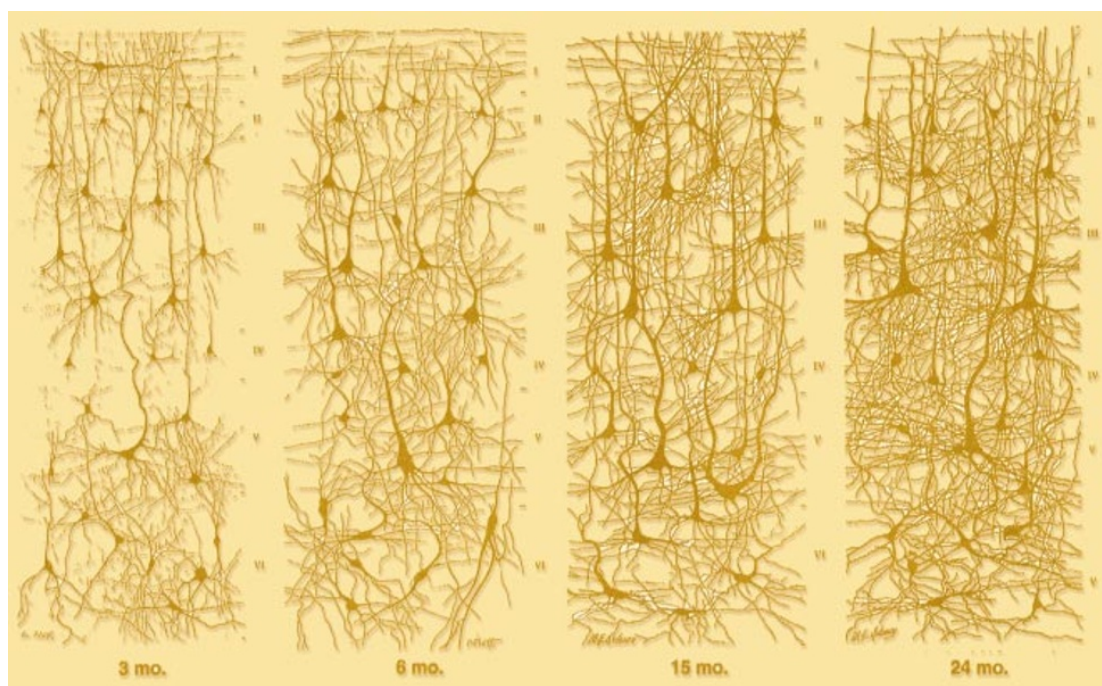

Growth factor: neurons develop rapidly in the human cortex between 3 and 24 months of age.

cognits from lower levels. In other words, the essential transactions underlying cognition involve the activation of different networks, arranged in hierarchies, that represent the building blocks of cognition. So, unlike Sherrington with his 'million-fold democracy' of neurons, Fuster proposes an isomorphism between the processes and the structures of mind and of the cerebral cortex.

This is a nice idea, but how well does it fit the facts? Fuster's chosen model of the parallel-distributed-processing network has been widely used, especially by cognitive psychologists. Such artificial neural networks have multiple layers, learn associatively, provide a complete output from incomplete input data, and show 'graceful' degradation in the face of partial damage. These are all presented as being satisfyingly brain-like.

But in truth, the performance of artificial neural networks is fragile in real-world environments. They seem ill-suited to representing Fuster's cognits, because the nodes of these networks usually represent functions with continuous values and their outputs combine with those of all other nodes to compute the output of the network. The nodes do not represent discrete bits that can simply be composed into well-defined higherorder constructs. Indeed, artificial neuronal networks can approximate any arbitrary nonlinear function without resorting to extensive hierarchies, yet hierarchies seem to be a fundamental organizing principle in brains. Artificial neural networks also scale notoriously badly, so most successful simulations have usually used networks with substantially fewer than 1,000 'neurons', in contrast to the 100,000 neurons contained in each cubic millimetre of neocortex. How we learn effectively and stably with such large networks is a mystery. Most models propose simple feedforward networks, which do not capture the essential recurrence and local processing of the neocortical circuits, nor do they capture the temporal aspect of processing, which is a feature of perception and action.

The capacity of the brain to excite itself is a fundamental necessity for cognition. And one of the key capacities that working memory provides is the possibility to delay a choice of action until the various options have been weighed up. This 'executive' model of prefrontal cortex has been popular since Phineas Gage survived a horrific mining accident in 1848 to become the first clinical exemplar of the consequences of damage to the prefrontal cortex. But as we 'ponder', 'deliberate' or 'examine' (all words deriving from Latin roots meaning 'to weigh'), can we find an analogous 'weighing' in the activity of neurons in our prefrontal cortex, or are the brain computations involved in these cognitive processes carried out by symbolic manipulations reminiscent of our use of numbers and letters? Exactly how do neurons make up one's mind?

Horace Barlow once proposed that the rate of neuronal activity reflects the probability of a certain stimulus being present. Decision-making requires that these probabilities be weighed, and one intriguing recent suggestion, by Michael N. Shadlen, is that this occurs in a second stage of processing in which sensory responses are accumulated from pools of neurons over time to form a single quantity, for example the logarithm of the likelihood ratio. The neurons that best 
fulfil this role are none other than those discovered by Fuster and Niki. If their persistent activity in the absence of a sensory cue is indeed the step of calculating a single decision variable based on information from several sources, then neurophysiologists have actually watched neurons making up the monkey's mind. What determines the moment of decision is not yet known, but just as 'decide' once meant to cut off, or bring to an end, so these neurons do indeed stop their activity when the decision is made.

There is a strong argument that we have made such great progress in understanding the neural basis of cognition only because neurons, and the networks that they form, compute in an analogue style. We can get an idea of the underlying computations by measuring the activity of single neurons, or the strength of the functional magnetic resonance imaging signal. It seems fantastic, but Fuster's progress report dares us to believe that the patterns woven by Sherrington's "enchanted loom", the cerebral cortex, are now well on the way to being understood.

Kevan Martin is at the Institute of

Neuroinformatics, University of Zurich/ETH, Winterthurerstrasse 190, 8057 Zurich, Switzerland.

\section{Suffocated or shot?}

\section{When Life Nearly Died: The Greatest Mass Extinction of \\ All Time}

by Michael Benton

Thames and Hudson: 2003. 336pp.

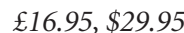

\section{Peter J. Bowler}

Whatever hit the Earth at the end of the Permian period certainly struck hard, killing $90 \%$ of living species. Compared with this, the extinction at the end of the Cretaceous period was comparatively minor, with only a $50 \%$ death rate. Yet the latter event is much better known, because among that 50\% were the last of the dinosaurs. Partly for this reason, Michael Benton uses the event at the end of the Cretaceous as an introduction to his account of the Permian extinction - he wants us to realize how limited it was in comparison with what he intends to describe.

But there is a deeper reason for linking the two episodes: Benton wants to show us how the catastrophist perspective has re-emerged in modern geology and palaeontology. $\mathrm{He}$ argues that the theory of catastrophic mass extinctions was widely accepted in the early nineteenth century, but was then driven underground by the gradualist perspective of Charles Lyell's uniformitarian geology and Darwin's theory of evolution. Only in the 1970s was catastrophism revived, through the claim that the dinosaurs were wiped out when an asteroid hit the Earth. Benton shows

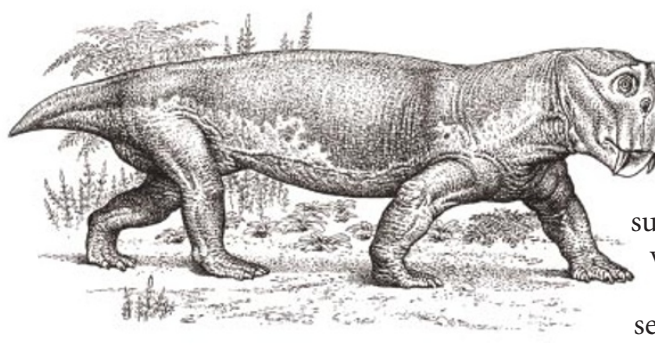

But such ideas went out of fashion in the mid-nineteenth century, and later catastrophists, including Murchison, favoured explanations based on the supposedly more intense geological activity in the young Earth. The asteroidimpact theory of dinosaur extinctions seems to parallel some of the earliest

Exit stage right - even though Lystrosaurus survived the extinction at the end of the Permian.

us how in the 1990s the evidence began to emerge that the species replacements marking the Permian-Triassic transition were also sudden, and hence were probably caused by some environmental trauma. He is describing both a geologically sudden event and a rapid transformation in our ideas about the Earth's past.

As a result, the book is partly historical in nature. It describes how the British geologist R. I. Murchison (himself a catastrophist) defined the Permian rocks of Russia in about 1840, and how Lyell and Darwin challenged the idea of mass extinctions by arguing that apparently sudden transitions in the fossil record were the result of gaps in the evidence, which created illusory jumps between one system of rocks and the next.

The triumph of darwinism ensured that catastrophist explanations were marginalized until they were revived by the asteroidimpact theory for the end of the Cretaceous. Even then, many palaeontologists resisted, arguing that the dinosaurs were declining anyway, so the impact only finished a job that had already been started by gradual environmental changes. At the time, knowledge of the Permian-Triassic transition was so limited that gradualism still seemed plausible here, too. Benton provides a graphic account of how more recent evidence has piled up, including his own experiences fossil hunting in Russia, making a catastrophic explanation inescapable.

There is one important twist in the story, however: Benton finds little support for the possibility that the Permian extinction was caused by an extraterrestrial agent. Wild theories about periodic bombardments by asteroids have not stood the test of time: the Permian event was probably triggered by massive volcanism, which injected poisonous gases into the atmosphere, both directly and by triggering the release of methane from deep-sea hydrates. Some geologists think that volcanism also played a role at the end of the Cretaceous. Significantly, Benton concludes by considering the implications of the latest, man-made mass extinction, asking what light the earlier events can throw on the potential for survival of modern species.

The historical aspect of Benton's book raises some intriguing questions. Many early catastrophists postulated the involvement of extraterrestrial agents - a comet was sometimes invoked as the cause of Noah's flood. speculations, but Benton has redressed the balance by favouring internal causes.

My one criticism of his account is that he accepts too readily the assumption that Lyell and Darwin marginalized all support for discontinuity in the Earth's history. There were few outright catastrophists left by around 1900, but many still believed that the history of life had been punctuated by environmental transitions far more rapid than anything observed in the recent past.

The real triumph of gradualism came with the modern darwinian synthesis of the mid-twentieth century, and even then it was confined to the English-speaking world. Benton notes that British and US palaeontologists of the 1950s ignored the catastrophism of Otto Schindewolf. But we need to recognize that German palaeontologists such as Schindewolf were continuing a long-standing tradition that had proved far more robust than our modern, Darwincentred histories acknowledge. The fact that modern catastrophists do not see a link back to that tradition tells us about the effectiveness of the neo-lyellian interlude of the mid-twentieth century.

Peter J. Bowler is in the Department of Social Anthropology, Queen's University Belfast, Belfast BT7 1NN, UK

\section{Hooke, life and thinker}

London's Leonardo: The Life and Work of Robert Hooke

by Jim Bennett, Michael Cooper,

Michael Hunter \& Lisa Jardine

Oxford University Press: 2003. 240 pp.

$£ 20, \$ 35$

\section{David R. Oldroyd}

Some devotees of Robert Hooke have regarded him as Britain's greatest scientific genius of the seventeenth century, the range of his interests and achievements being hard to conceive. He is a fruitful subject for historical enquiry as he left behind him a large archival trail, and, with his polymathic interests, he has attracted much attention. A good general overview, Robert Hooke by Margaret 'Espinasse (Heinemann), was published in 1956. Since then, studies of Hooke have expanded greatly to the point where we have a detailed knowledge of the man, although not all within the pages of a 